\title{
Relationships amongst some bacterial and yeast lactate and mandelate dehydrogenases
}

\author{
Charles A. Fewson, ${ }^{1 *}$ Darren P. Baker, ${ }^{1} \uparrow$ Ronald M. Chalmers, ${ }^{1} \S$ Jeffrey N. Keen, ${ }^{2}$ \\ Ian D. Hamilton, ${ }^{1}$ Alan J. Scott ${ }^{1}$ and Muhammad Yasin ${ }^{1}$ \\ ${ }^{1}$ Department of Biochemistry, University of Glasgow, Glasgow G12 8QQ, UK \\ ${ }^{2}$ Department of Biochemistry and Molecular Biology, University of Leeds, Leeds LS2 9JT, UK
}

(Received 15 October 1992; revised 15 January 1993; accepted 4 February 1993)

\begin{abstract}
Five yeast strains were isolated by enrichment culture on the basis of their ability to grow on mandelate and two of these strains were identified as Rhodotorula glutinis. In addition, a range of yeasts from culture collections was screened for growth on mandelate. The results suggest that mandelate utilization is a widespread but not universal characteristic within the genus Rhodotorula. Several of the yeasts contained an inducible NAD-dependent $D(-)$-mandelate dehydrogenase and an inducible dye-linked (presumably flavoprotein) $L(+)$-mandelate dehydrogenase. All the $\mathrm{D}(-)$-mandelate dehydrogenases from the yeasts showed immunological cross-reactivity with each other (as judged by both immunoinhibition and immunoblotting), as did all the yeast $\mathrm{L}(+)$-mandelate dehydrogenases that were tested. Determination of $\mathrm{N}$-terminal amino acid sequences of several bacterial and yeast lactate and mandelate dehydrogenases, together with the evidence from the immunological studies, confirmed and extended previous proposals that there are several major groups of such dehydrogenases: FMN-dependent, membrane-bound $\mathbf{L}(+)$-lactate and $\mathbf{L}(+)$-mandelate dehydrogenases $\left(M_{\mathrm{r}}=\right.$ approx. 44000) in bacteria, mitochondrial flavocytochrome $b_{2} L(+)$-lactate and $L(+)$-mandelate dehydrogenases $\left(M_{\mathrm{r}}=\right.$ approx. 59000) in yeasts, FAD-dependent, membrane-bound $\mathrm{D}(-)$-lactate and $\mathrm{D}(-)$-mandelate dehydrogenases in bacteria, and soluble NAD-dependent $\mathrm{D}(-)$-mandelate dehydrogenases in both bacteria and yeasts.
\end{abstract}

\section{Introduction}

A wide range of bacteria can use mandelate for growth and the first metabolic step is generally a stereospecific dehydrogenation (Fewson, 1988). There seem to be several different types of mandelate dehydrogenases, depending on the enantiomer that is oxidized and on the organism (Fewson, 1992). The gene encoding $\mathrm{L}(+)$ mandelate dehydrogenase in Pseudomonas putida has been sequenced and the enzyme has regions of sequence similarity to the FMN-containing enzyme glycolate oxidase from spinach and to the FMN-domain of yeast flavocytochrome $b_{2} \mathrm{~L}(+)$-lactate dehydrogenase (Tsou et al., 1990). The $\mathrm{L}(+)$-mandelate dehydrogenase from Acinetobacter calcoaceticus contains non-covalently bound FMN as cofactor and has a very similar $M_{\mathrm{r}}$

${ }^{*}$ Author for correspondence. Tel. 0413398855 ; fax 0413304620.

$\dagger$ Present address: Department of Chemistry, Boston College, MA 02167 , USA.

$\S$ Present address: Department of Biochemistry and Molecular Biology, Harvard University, Cambridge, MA 02138, USA. (approx. 44000) to the enzyme from $P$. putida (Hoey et al., 1987). The $\mathrm{L}(+)$-mandelate dehydrogenase and the $\mathrm{L}(+)$-lactate dehydrogenase from $A$. calcoaceticus are very similar to each other and to the $\mathrm{L}(+)$-lactate dehydrogenase from Escherichia coli (Hoey et al., 1987). However, the $\mathrm{D}(-)$-mandelate dehydrogenase from $A$. calcoaceticus is larger $\left(M_{\mathrm{r}}=\right.$ approx. 60000) and contains FAD as cofactor (Allison et al., 1985). Overall, the $\mathrm{D}(-)$ mandelate dehydrogenase and $\mathrm{D}(-)$-lactate dehydrogenase from $A$. calcoaceticus are similar to each other and to the $\mathrm{D}(-)$-lactate dehydrogenase from $E$. coli (Allison et al., 1985). All these mandelate and lactate dehydrogenases are membrane-bound enzymes.

The situation in the yeast Rhodotorula graminis is rather different. The $\mathrm{D}(-)$-mandelate dehydrogenase is a soluble, NAD-dependent enzyme (Baker \& Fewson, 1989; Durham, 1984; Durham et al., 1984) which has at least superficial resemblance to the NAD-dependent $\mathrm{D}(-)$-mandelate dehydrogenase from Lactobacillus curvatus (Hummel et al., 1988) and to the NADdependent $\mathrm{D}(-)$-2-hydroxyisocaproate dehydrogenase from Streptococcus faecalis that can use $\mathrm{D}(-)$-mandelate as substrate (Yamazaki \& Maeda, 1986). The L(+)- 
mandelate dehydrogenase from $R$. graminis, on the other hand, is a flavocytochrome $b$, that appears to be very like the $\mathrm{L}(+)$-lactate dehydrogenases from yeasts such as Saccharomyces cerevisiae and Hansenula anomala (Chapman et al., 1991; Yasin \& Fewson, 1993).

The picture that emerges, therefore, is that mandelate dehydrogenases, like lactate dehydrogenases (Garvie, 1980), are stereospecific enzymes that can be either NAD(P)-dependent or flavoproteins and the latter type may or may not contain haem. The similarity among certain of these dehydrogenases is perhaps not too surprising because their substrates are all 2-hydroxycarboxylic acids, in which the methyl group of lactate is replaced by a benzene ring in mandelate. The overall similarity between lactate and mandelate dehydrogenases, supported by the much more convincing sequence evidence for the relationship between $\mathrm{L}(+)$ mandelate dehydrogenase from $P$. putida and certain other enzymes (Tsou et al., 1990), has led to the suggestion that mandelate dehydrogenases probably arose by the duplication and mutation of genes for other 2-hydroxyacid dehydrogenases (Tsou et al., 1990; Fewson, 1992). The similarities within certain sub-groups of these dehydrogenases (e.g. the $\mathrm{D}$ - and the L-isomerspecific flavoproteins or the NAD-dependent soluble dehydrogenases) support the notion that they are related evolutionarily. We set out to gain more general evidence for or against this hypothesis by examining mandelate dehydrogenases from a range of organisms. The bulk of work on mandelate metabolism has been done with bacteria, and rather few eukaryotic enzymes were available for study. Although some filamentous fungi can oxidize mandelate, perhaps by co-metabolism, the activities of the mandelate dehydrogenases are extremely low (Jamaluddin et al., 1970; Ramanarayanan \& Vaidyanathan, 1973; Kishore et al., 1976; Ramakrishna Rao \& Vaidyanathan, 1977) and we have not been able to study them in detail (A. J. Scott and C. A. Fewson, unpublished observations). The first aim of this work was therefore to obtain a range of fungi, preferably yeasts, that contained mandelate dehydrogenases with sufficiently high activity that they could be studied further. The second aim was to compare a range of bacterial and yeast lactate and mandelate dehydrogenases by two complementary approaches: immunological cross-reactions and $\mathrm{N}$-terminal amino acid sequencing.

\section{Methods}

Maintenance, growth and harvesting of micro-organisms and preparation of extracts. Strains of Acinetobacter calcoaceticus and Pseudomonas putida were as listed in Fewson et al. (1988) and Streptococcus faecalis IFO 12964 was obtained from the Institute of Fermentation, Osaka, Japan. Rhodotorula graminis KGX 39 was obtained from $\mathrm{Dr}$
D. R. Durham, Genex Corporation, Gaithersburg, MD 20877, USA, and other yeasts were from the Centraalbureau voor Schimmelcultures, Baarn, The Netherlands (CBS), the Czechoslovak Collection of Yeasts, Bratislava, Czechoslovakia (CCY), the Instituto Jaime Ferran de Microbiologia del Consejo Superior de Investigaciones Çientificas, Madrid, Spain (IJFM), the National Collection of Yeast Cultures, AFRC Institute of Food Research, Norwich, UK (NCYC) or the National Collection of Pathogenic Fungi, London, UK (NCPF).

Samples (approx. $1 \mathrm{~g}$ ) for enrichment of mandelate-utilizing yeasts were suspended in sterile distilled water $(10 \mathrm{ml})$, mixed and transferred to $50 \mathrm{ml}$ yeast nitrogen base (Difco) containing $5 \mathrm{mM}$-DL-mandelate and $100 \mu \mathrm{g}$ chloramphenicol $\mathrm{ml}^{-1}$. Samples that showed growth were serially transferred in the same medium and plated on nitrogen base/mandelate/chloramphenicol agar plates and incubated at $23^{\circ} \mathrm{C}$. Representative colonies were selected and inoculated into fresh selective media and onto Sabouraud-glucose agar, and grown at $23^{\circ} \mathrm{C}$. Two isolates (DB2 and $\mathrm{DB} 3$, isolated from rabbit faeces and garden compost, respectively) were pink budding yeasts that grew on solid media as smooth, shining, circular, raised colonies. Isolates DB2 and DB3 have been identified as strains of Rhodotorula glutinis at the NCYC where they have been deposited as strains NCYC 2439 and NCYC 2440, respectively. A third yeast (DB11, isolated from sheep faeces) was indistinguishable from the first two except by its darker colour. Two other isolates (DB10 from sheep faeces and DB18 from deer faeces) were both white/cream in colour and grew on solid media as smooth, shining, raised colonies.

Micro-organisms were generally maintained as described previously (Allison et al., 1985; Baker \& Fewson, 1989) and they were grown and harvested by conventional methods. Extracts were prepared by disruption with an ultrasonic probe (Fewson et al., 1988) or by passage through a French press (Baker \& Fewson, 1989). Experiments with the dye-linked (i.e. flavoprotein) dehydrogenases generally used extracts prepared in buffers containing $0.5 \mathrm{mg}$ Triton X-100 $\mathrm{ml}^{-1}$.

Preparation and assay of enzymes. NAD-dependent $\mathrm{D}(-)$-mandelate dehydrogenase and dye-dependent $\mathrm{L}(+)$-mandelate dehydrogenase from $R$. graminis KGX 39 were prepared by the methods of Baker \& Fewson (1989) and Yasin \& Fewson (1993), respectively. L(+)Mandelate dehydrogenase, $\mathrm{L}(+)$-lactate dehydrogenase, $\mathrm{D}(-)$-mandelate dehydrogenase and $\mathrm{D}(-)$-lactate dehydrogenase were purified from A. calcoaceticus strain $\mathrm{Cl} 219$ by the methods of Hoey et al. (1987), Allison et al. (1985) and Allison \& Fewson (1986). L(+)Mandelate dehydrogenase was purified from P. putida NCIB 9474 by a similar method to that used for the equivalent enzyme from $A$. calcoaceticus (Hoey et al., 1987). L(+)-Lactate dehydrogenase from Saccharomyces cerevisiae was obtained from Sigma. A crude extract of Lactobacillus curvatus DSM 20019 was kindly provided by Professor W. Hummel, Institute for Enzyme Technology, University of Düsseldorf, Germany.

Enzyme activities were measured as described previously (Baker \& Fewson, 1989; Yasin \& Fewson, 1993) except that in the experiments described in Table 1, bovine serum albumin and $N$-methylphenazonium methosulphate were omitted from the reaction mixture used to assay $\iota(+)$-mandelate dehydrogenase.

$N$-Terminal sequence analysis. D-(-)-Mandelate dehydrogenase from $R$. graminis was sequenced directly after purification and the other enzymes were separated on SDS-polyacrylamide gels, and then visualized by light staining with Coomassie Brilliant Blue and removed from the gels by the electroelution-electrodialysis method (Wilson \& Yuan, 1989). The $\mathrm{N}$-terminal sequences were determined by automated solid-phase Edman degradation as described previously (Chalmers et al., 1991).

Immunological methods. Antisera raised against $\mathrm{D}(-)$-mandelate dehydrogenase and SDS-denatured $\mathrm{L}(+)$-mandelate dehydrogenase 
Table 1. Mandelate dehydrogenase activities of various yeasts

The yeasts were grown, harvested, disrupted and their enzyme activities measured in extracts as described in Methods. The results are means of two experiments. ND, Not detectable.

\begin{tabular}{|c|c|c|c|c|}
\hline \multirow[b]{2}{*}{ Yeast } & \multicolumn{4}{|c|}{ Enzyme activity [nmol $\left.\mathrm{min}^{-1}(\mathrm{mg} \text { protein })^{-1}\right]$} \\
\hline & $\begin{array}{l}\mathrm{NAD}^{+} \text {-dependent } \\
\mathrm{D}(-) \text {-mandelate } \\
\text { dehydrogenase }\end{array}$ & $\begin{array}{l}\text { NADH-dependent } \\
\text { phenylglyoxylate } \\
\text { reductase }\end{array}$ & $\begin{array}{c}\text { NADPH-dependent } \\
\text { phenylglyoxylate } \\
\text { reductase }\end{array}$ & $\begin{array}{c}\text { Dye-linked } \\
\mathrm{L}(+) \text {-mandelate } \\
\text { dehydrogenase }\end{array}$ \\
\hline R. graminis KGX 39 & 440 & 2280 & ND & 80 \\
\hline R. graminis NCYC 980 & 30 & 1090 & ND & 30 \\
\hline R. graminis NCYC 1401 & ND & 170 & 10 & 10 \\
\hline R. glutinis NCYC 59 & ND & ND & ND & 70 \\
\hline R. glutinis NCYC 2439 & 200 & 1090 & 40 & 30 \\
\hline R. glutinis NCYC 2440 & 130 & 760 & 40 & 30 \\
\hline R. grinbergsii IJFM 6000 & ND & ND & ND & 8 \\
\hline
\end{tabular}

from $A$. calcoaceticus were those obtained in previous work (Fewson $e t$ al., 1988; Hoey \& Fewson, 1990) and antisera against L(+)-mandelate dehydrogenase and $\mathrm{D}(-)$-mandelate dehydrogenase from $R$. graminis KGX 39 were prepared using similar techniques. Immunoinhibition and immunoblotting experiments were done as described previously (Fewson et al., 1988; Chalmers et al., 1991).

\section{Results and Discussion}

\section{Growth of yeasts on DL-mandelate}

Of the 34 yeast strains tested, which represented at least 28 species and 17 different genera, 11 could grow on DLmandelate. Of these, eight were strains of Rhodotorula and although the other three were of unknown identity, one of them (DB11) was pink in colour and is probably a member of this genus. Strains that grew on DLmandelate were: $R$. glutinis NCYC 59, NCYC 2439 and NCYC 2440; $R$. graminis strains KGX 39, NCYC 980 and NCYC 1401; $R$. grinbergsii IJFM $6000 ; R$. rubra NCYC 758; and isolates DB10, DB11 and DB18. Strains that could not grow on DL-mandelate were: Candida nitratophila NCYC 556, C. pseudotropicalis NCPF 3234, C. tropicalis NCYC 1503, C. utilis NCYC 321, Debaryomyces hansenii CBS 767, Endomycopsis capsularis CBS 2519, Hanseniaspora valbyensis CBS 479, Hansenula anomala NCYC 432, Lodderomyces elongisporus NCYC 928, Pachysolen tannophilus NCYC 614, Pichia membranaefaciens CBS 107, Rhodosporidium toruloides NCYC 819, Rhodotorula graminis NCYC 502, R. minuta NCYC 931, $R$. nothofagi CCY 20-19-1, $R$. pilimanae NCYC 759, Saccharomyces bayanus CBS 380, Sporobolomyces roseus NCYC 1609, Torulopsis colliculosa CBS 133, Trichosporon cutaneum CBS 2466, Trigonopsis variabilis CBS 1040, Wickerhamia fluorescens CBS 4565 and Wingia robertsii CBS 2934.

Hegeman et al. (1970) mentioned a yeast that could grow on $\mathrm{L}(+)$-mandelate and it is clear that it is easy to use selective enrichment to isolate yeasts that can use mandelate as the sole source of carbon and energy for growth. Nevertheless, mandelate utilization is not a very widespread characteristic of yeasts. It seems likely that the ability to utilize mandelate is a common but not universal trait of the genus Rhodotorula; however, as with bacteria such as $A$. calcoaceticus and $P$. putida, it is a strain-specific rather than a species-specific characteristic (Fewson, 1988).

\section{Mandelate dehydrogenase activities in yeasts}

Mandelate dehydrogenase activity was found in seven of the 11 yeasts that could grow on mandelate (Table 1) but was not detected in isolates DB10, DB11, DB18 or $R$. rubra NCYC 758, all strains that grew rather slowly. All of the seven strains had a dye-linked $\mathrm{L}(+)$-mandelate dehydrogenase. $\mathrm{NAD}^{+}$-dependent $\mathrm{D}(-)$-mandelate dehydrogenase activity was found in $R$. graminis strains KGX 39 and NCYC 980 and in R. glutinis strains NCYC 2439 and 2440 , and could be measured by the oxidation of $\mathrm{D}(-)$-mandelate with $\mathrm{NAD}^{+}$as well as by the reduction of phenylglyoxylate by NADH. However, in $R$. graminis NCYC 1401 only the reduction of phenylglyoxylate could be measured, presumably because the low specific activity of $\mathrm{D}(-)$-mandelate dehydrogenase could be detected only using the more sensitive reverse assay (Baker \& Fewson, 1989). A previously unreported NADPH-dependent phenylglyoxylate reductase activity was found in three strains; however, the specific activity was low and the enantiomeric form of the product was not determined. None of the strains contained detectable activities of NAD ${ }^{+}$-dependent $\mathrm{L}(+)$-mandelate dehydrogenase, $\mathrm{NADP}^{+}$-dependent $\mathrm{L}(+)$ - or $\mathrm{D}(-)$-mandelate dehydrogenase or dye-linked $\mathrm{D}(-)$-mandelate dehydrogenase. The inability to detect mandelate dehydrogenase activity of any sort in $R$. rubra NCYC 758 or in isolates DB10, DB11 or DB18 may be because the enzymes are present but at very low levels, or because we were not using the optimum conditions to measure them; alterna- 
Table 2. Immunoinhibition of mandelate dehydrogenases in various yeasts caused by antisera raised against the $D(-)$ - and $L(+)$-mandelate dehydrogenases from $R$. graminis $K G X 39$

Extracts of the four yeasts were incubated for $1 \mathrm{~h}$ on ice with various dilutions of sera prepared from rabbits immunized with either $\mathrm{L}(+)$-mandelate dehydrogenase or $\mathrm{D}(-)$-mandelate dehydrogenase purified from $R$. graminis $\mathrm{KGX} 39$ and the enzyme activities were then measured. Graphs of remaining enzyme activity against dilution of antiserum were drawn and the dilutions of antisera required to give $50 \%$ inhibition of enzyme activity were calculated. The results are ranges for several experiments.

\begin{tabular}{|c|c|c|c|c|}
\hline \multirow[b]{3}{*}{ Rabbit no.: } & \multicolumn{4}{|c|}{ Dilution of antiserum required to give $50 \%$ inhibition of enzyme activity } \\
\hline & \multicolumn{2}{|c|}{$\mathrm{L}(+)$-Mandelate dehydrogenase } & \multicolumn{2}{|c|}{$\mathrm{D}(-)$-Mandelate dehydrogenase } \\
\hline & 1153 & 1154 & 253 & 319 \\
\hline R. graminis KGX 39 & $1: 4-1: 16$ & $1: 16-1: 64$ & $1: 256-1: 512$ & $1: 64-1: 128$ \\
\hline R. graminis NCYC 980 & $1: 2-1: 4$ & $1: 16-1: 32$ & $1: 16-1: 32$ & $1: 8-1: 16$ \\
\hline R. glutinis NCYC 2439 & $1: 1-1: 2$ & $1: 16-1: 32$ & $1: 16-1: 32$ & $1: 32-1: 64$ \\
\hline R. glutinis NCYC 2440 & $1: 1-1: 2$ & $1: 16-1: 32$ & $1: 16-1: 32$ & $1: 16-1: 32$ \\
\hline
\end{tabular}

(a)

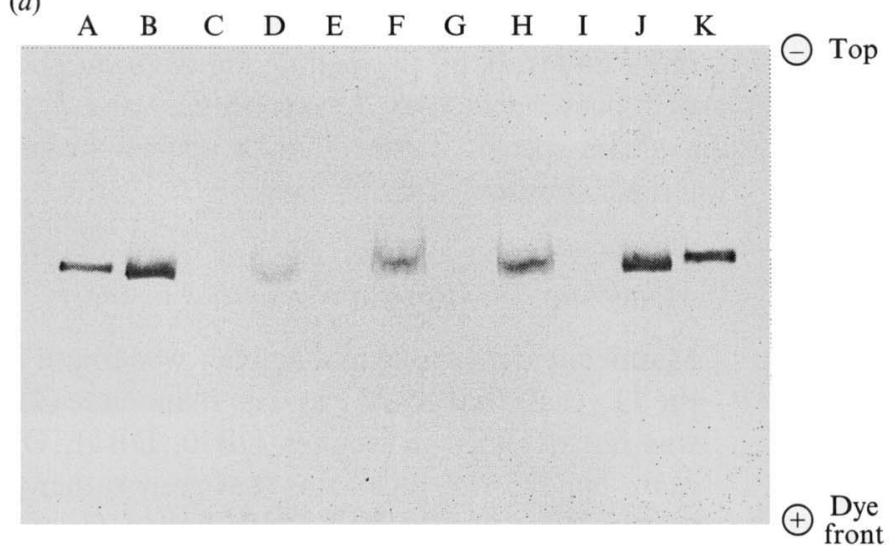

(b)

$\begin{array}{llllllllllllllll}\text { A } & \text { B } & \text { C } & \text { D } & \text { E } & \text { F } & \text { G } & \text { H } & \text { I }\end{array}$

Fig. 1. Immunoblots of various yeast mandelate dehydrogenases probed with antisera raised against $L(+)$-mandelate and $\mathrm{D}(-)$ mandelate dehydrogenases from R. graminis KGX 39. Purified enzymes as well as extracts of yeasts that had been grown on glucose or mandelate were electrophoresed and then the proteins electroblotted onto nitrocellulose, probed with the appropriate antiserum and developed. (a) Blot probed with serum raised against $\mathrm{L}(+)$-mandelate dehydrogenase. The tracks that were electrophoresed contained: A and K, pure L(+)-mandelate dehydrogenase from R. graminis KGX 39 (33 ng); B and J, extract of $R$. graminis grown on mandelate $(23 \mu \mathrm{g})$; I, extract of $R$. graminis grown on glucose $(23 \mu \mathrm{g})$; C, extract of $R$. graminis NCYC 980 grown on glucose (50 $\mu \mathrm{g})$; D, extract of $R$. graminis NCYC 980 grown on mandelate $(50 \mu \mathrm{g}) ; \mathrm{E}$, extract of $R$. glutinis NCYC 2439 grown on glucose $(36 \mu \mathrm{g}) ; \mathrm{F}$, extract of $R$. glutinis NCYC 2439 grown on mandelate $(36 \mu \mathrm{g}) ; \mathrm{G}$, extract of $R$. glutinis NCYC 2440 grown on glucose $(34 \mu \mathrm{g}) ; \mathrm{H}$, extract of $R$. glutinis NCYC 2440 grown on mandelate $(34 \mu \mathrm{g})$. (b) Blot probed with serum raised against $\mathrm{D}(-)$-mandelate dehydrogenase. The tracks that were electrophoresed contained: A, pure $\mathrm{D}(-)$-mandelate dehydrogenase (500 $\mathrm{ng}$ ); B, extract of $R$. graminis $\mathrm{KGX} 39$ grown on mandelate $(21 \mu \mathrm{g})$; C, extract of $R$. graminis KGX 39 grown on glucose $(21 \mu \mathrm{g}) ; \mathrm{D}$, extract of $R$. glutinis NCYC 2439 grown on mandelate $(41 \mu \mathrm{g})$; E, extract of $R$. glutinis NCYC 2439 grown on glucose $(41 \mu \mathrm{g}) ; \mathrm{F}$, extract of $R$. glutinis NCYC 2440 grown on mandelate $(50 \mu \mathrm{g}) ; \mathrm{G}$, extract of $R$. glutinis NCYC 2440 grown on glucose $(50 \mu \mathrm{g}) ; \mathrm{H}$, extract of $R$. graminis $\mathrm{NCYC} 980$ grown on mandelate $(50 \mu \mathrm{g})$; I, extract of $R$. graminis NCYC 980 grown on glucose $(50 \mu \mathrm{g})$.

tively, mandelate metabolism in these strains may not be initiated by dehydrogenation.

\section{Immunological cross-reactivities}

The sera prepared by immunization of rabbits with the dye-linked D(-)-mandelate dehydrogenase of $A$. calcoaceticus, the dye-linked $\mathrm{L}(+)$-mandelate dehydrogenase of $R$. graminis KGX 39 and the NAD-dependent $\mathrm{D}(-)$ - mandelate dehydrogenase of $R$. graminis KGX 39 all showed substantial inhibition when they were incubated with the native enzymes which had been used as antigens. There was no inhibition by pre-immune sera. The only cross-reactions were those between antisera raised against the two yeast enzymes and the analogous native enzymes from the other yeasts that had sufficient enzyme activity to be tested (Table 2). In addition, serum from rabbit 1154 (but not that from rabbit 1153) immunized 
A

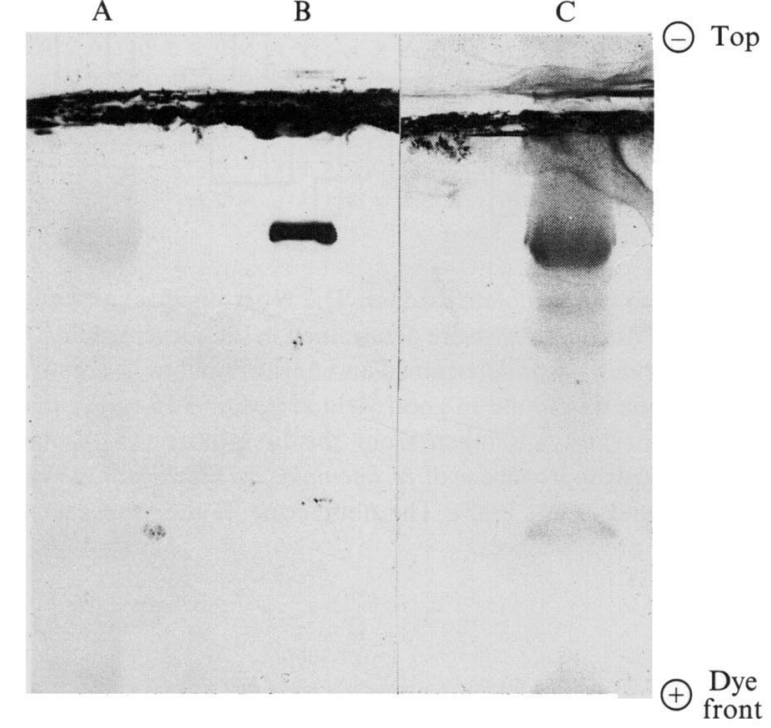

when large amounts of protein were loaded on the gel (Fig. 2).

There appears to be a rule of thumb that immunological cross-reactions are detectable between native antigens that have greater than about $60 \%$ sequence identity and between denatured antigens that have greater than about $40 \%$ sequence identity (see references in Chalmers et al., 1991). If this holds for these lactate and mandelate dehydrogenases, then it would seem from the strong cross-reactions in both immunoinhibition experiments (Table 2 ; presumably chiefly testing native configurations) and immunoblots (Fig. 1; presumably chiefly concerned with denatured configurations) that the $\mathrm{L}(+)$-mandelate dehydrogenase from $R$. graminis KGX 39 shares considerably more than $60 \%$ overall sequence identity with the $\mathrm{L}(+)$-mandelate dehydrogenases from $R$. graminis NCYC 980 and $R$. glutinis strains NCYC 2439 and 2440; the D(-)-mandelate dehydrogenases of these organisms are also closely related. In addition, barely detectable cross-reactions between $\mathrm{L}(+)$-mandelate dehydrogenase from $R$. graminis KGX 39 and $\mathrm{L}(+)$-lactate dehydrogenase from Sacc. cerevisiae (results not shown) and between the $\mathrm{L}(+)$-mandelate dehydrogenases from $A$. calcoaceticus and $P$. putida (Fig. 2) suggest that these two pairs of enzymes have some sequence identity, a result which is consistent with their $\mathrm{N}$-terminal amino acid sequences (described below).

In other experiments (results not shown) we failed to find any reaction of the antiserum against $D(-)$ mandelate dehydrogenase from $R$. graminis KGX 39 with either the $\mathrm{D}(-)$-mandelate dehydrogenase from $L$. curvatus or the $\mathrm{D}(-)$-2-hydroxyisocaproate dehydrogenase from Strep. faecalis; however, this does not necessarily mean that these enzymes are not homologous, but it may simply reflect the taxonomic remoteness of the organisms resulting in insufficient sequence identity to be detected by immunological methods even if the enzymes are derived from common ancestors.

immunoblots with their homologous antigens. In the case of immunoblotting, there was no indication of cross-reaction between the antiserum raised against $\mathrm{L}(+)$-mandelate dehydrogenase from $R$. graminis $\mathrm{KGX}$ 39 and the $\mathrm{L}(+)$-lactate dehydrogenase from Sacc. cerevisiae (results not shown). The immunoblots did, however, show two significant sets of cross-reactivity. First, all the yeast $\mathrm{L}(+)$ - and $\mathrm{D}(-)$-mandelate dehydrogenases that were tested were detected by the antiserum raised against the analogous enzymes from $R$. graminis KGX 39 (Fig. 1). This experiment also demonstrates that the mandelate dehydrogenases that were tested are inducible. Secondly, $L(+)$-mandelate dehydrogenase from $P$. putida showed a very weak cross-reaction with the antiserum against $\mathrm{L}(+)$-mandelate dehydrogenase from $A$. calcoaceticus, but this could be detected only

\section{$N$-Terminal amino acid sequences}

(i) Dye-linked $L(+)$-lactate and $L(+)$-mandelate dehydrogenases

Tsou et al. (1990) discovered that $\mathrm{L}(+)$-mandelate dehydrogenase from $P$. putida has extensive positional identity with the FMN-containing enzyme glycolate oxidase and with the FMN-containing domain of $\mathrm{L}(+)$ lactate dehydrogenase from Sacc. cerevisiae, which in turn is homologous with the equivalent domain from the $\mathrm{L}(+)$-lactate dehydrogenase from $H$. anomala (Chapman et al., 1991). From the present work (Fig. 3) it is clear that the $\mathrm{L}(+)$-mandelate dehydrogenases from $A$. calcoaceticus and $P$. putida are homologous with each other 


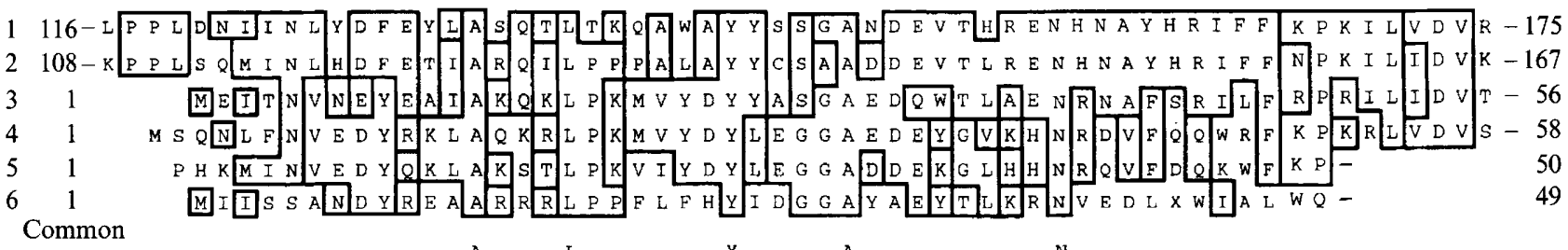

Fig. 3. Sequence alignments of $\mathrm{L}(+)$-lactate and $\mathrm{L}(+)$-mandelate dehydrogenases and glycolate oxidase. The $\mathrm{N}$-terminal sequences of $\mathrm{L}(+)$-mandelate dehydrogenase (5) and L (+)-lactate dehydrogenase (6) from A. calcoaceticus were determined in the present study. In addition, the $\mathrm{N}$-terminal sequence of $\mathrm{L}(+)$-mandelate dehydrogenase from $P$. putida (4) was determined and it was found to be identical with that deduced from the DNA sequence (Tsou et al., 1990) except that alanine was found in good yield at position 15 rather than arginine as in the deduced sequence. The sequences for the other three enzymes are taken from the literature: $L(+)$-lactate dehydrogenase of Sacc. cerevisiae (1) (Guiard, 1985; Lederer, 1985); L(+)-lactate dehydrogenase of H. anomala (2) (Black et al., 1989); and glycolate oxidase from spinach (3) (Volokita \& Somerville, 1987; Cederlund et al., 1988). The numbering of the yeast lactate dehydrogenases is based on the mature protein sequences after removal of the pre-sequences.

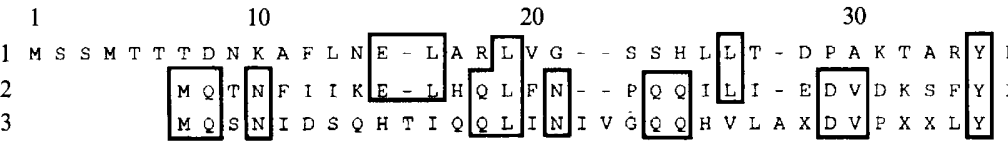

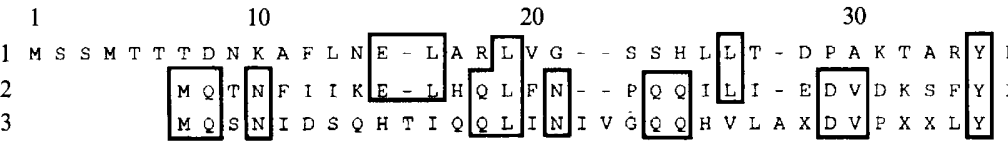

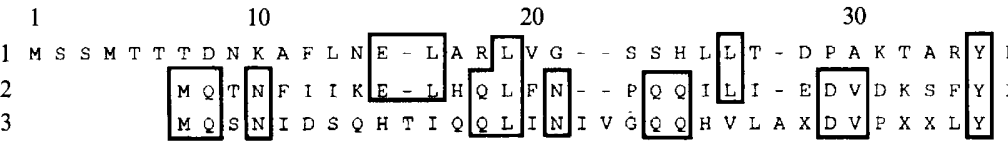

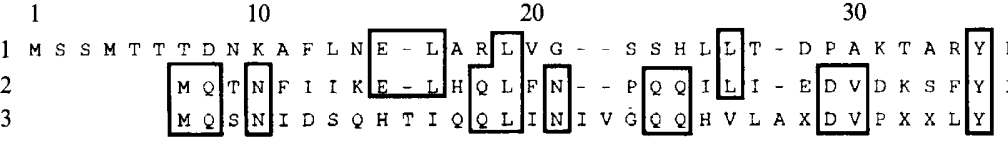
40

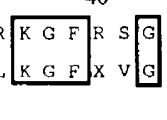

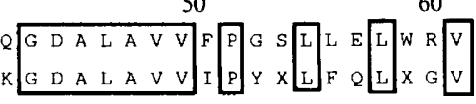

Fig. 4. Sequence alignments of $\mathrm{D}(-)$-lactate and $\mathrm{D}(-)$-mandelate dehydrogenases. The $\mathrm{N}$-terminal sequences of $\mathrm{D}(-)$-mandelate dehydrogenase (2) and $\mathrm{D}(-)$-lactate dehydrogenase (3) from $A$. calcoaceticus were determined in the present study and that for $\mathrm{D}(-)$ lactate dehydrogenase from $E$. coli (1) was deduced from the DNA sequence (Campbell et al., 1984; Rule et al., 1985).

(62\% positional identity for the 50 residues at their $\mathrm{N}-$ termini) and with the $\mathrm{L}(+)$-lactate dehydrogenase from A. calcoaceticus, another FMN-containing enzyme of similar $M_{\mathrm{r}}$ (Allison \& Fewson, 1986; Hoey et al., 1987).

(ii) Dye-linked $D(-)$-lactate and $D(-)$-mandelate dehydrogenases

D( - )-Mandelate dehydrogenase from A. calcoaceticus shares significant positional identity (33 and $36 \%$, respectively) with the $\mathrm{D}(-)$-lactate dehydrogenases from both $A$. calcoaceticus and $E$. coli (Fig. 4). All three enzymes contain FAD and have a similar $M_{\mathrm{r}}$ (Allison et al., 1985). Perhaps surprisingly, the two lactate dehydrogenases are not very similar over the sequences examined and it is unfortunate that repeated attempts failed to extend the sequence of the enzyme from $A$. calcoaceticus.

(iii) $N A D(P)$-dependent $D(-)$-mandelate dehydrogenases Although approximately one-third of the sequence of the NAD-dependent $\mathrm{D}(-)$-mandelate dehydrogenase from $R$. graminis has been determined (D. P. Baker, J. N. Keen and J.S. Miles, unpublished results), no obvious similarities have been observed with the sequences of other soluble D-isomer-specific 2-hydroxyacid dehydrogenases (Taguchi \& Ohta, 1991) or with any other enzymes in the literature or databases. Moreover, the consensus nucleotide-binding sequence GXGXXG, which is highly conserved in numerous NAD-dependent oxidoreductases, has not yet been found in the $\mathrm{D}(-)$ mandelate dehydrogenase sequence. Unfortunately, no sequence information appears to be available for either $\mathrm{D}(-)$-mandelate dehydrogenase from $L$. curvatus or $\mathrm{D}(-)$-hydroxyisocaproate dehydrogenase from Strep. faecalis (which has mandelate dehydrogenase activity; see Introduction) but the $\mathrm{D}(-)$-hydroxyisocaproate dehydrogenase from $L$. casei is homologous with other D-isomer-specific 2-hydroxyacid dehydrogenases (Lerch et al., 1989; Taguchi \& Ohta, 1991).

\section{Conclusions}

When account is taken of the results in the present paper and related work, it can be concluded that:

1. The ability to utilize mandelate is quite common in yeasts of the genus Rhodotorula. At least four independently-isolated strains possess NAD-linked $\mathrm{D}(-)$ mandelate dehydrogenases that are probably homologous with each other. They also possess $\mathrm{NAD}(\mathrm{P})$ independent flavocytochrome $b \quad \mathrm{~L}(+)$-mandelate dehydrogenases that are probably homologous both with each other and with $\mathrm{L}(+)$-lactate dehydrogenases from the yeasts Sacc. cerevisiae and $H$. anomala.

2. The $\mathrm{L}(+)$-lactate and $\mathrm{L}(+)$-mandelate dehydrogenases of the yeasts are two-domain enzymes in which the $\mathrm{N}$-terminal haem-containing domain is homologous with mammalian cytochrome $b_{5}$ and in which the FMNcontaining domain is homologous with certain bacterial $\mathrm{L}(+)$-mandelate and $\mathrm{L}(+)$-lactate dehydrogenases (Fig. 5 ) and with some 2-hydroxyacid oxidases. Whether there 


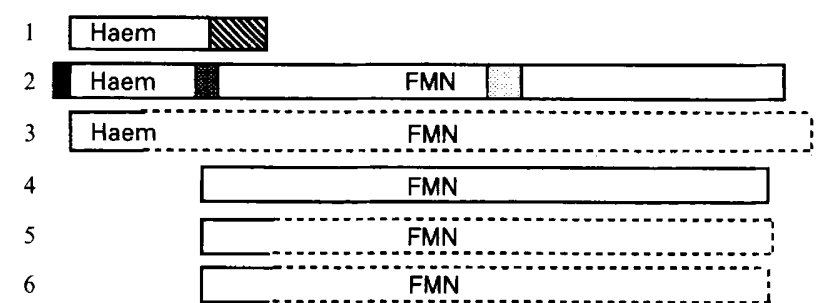

Fig. 5. Diagrammatic representation of bacterial and yeast $\mathrm{L}(+)$-lactate and $\mathbf{L}(+)$-mandelate dehydrogenases showing the domain structures: pre-sequence, black; hydrophobic tail, hatched; hinge region, stippled; proteinase-sensitive loop, grey. Solid line bars represent proteins for which the entire DNA sequence is known. The solid line in dashed line bars represents the extent of the $\mathrm{N}$-terminal residues sequenced. The lengths of the bars are approximately proportional to the $M_{\mathrm{r}}$ values and sequences showing identity are aligned. The information is taken from the present work, Chapman et al. (1991), Fewson (1992), Tsou et al. (1990) and Yasin \& Fewson (1993). 1, Beef microsomal cytochrome $b_{5}$; 2, Sacc. cerevisiae $\mathrm{L}(+)$-lactate dehydrogenase; $3, R$. graminis $\mathrm{L}(+)$ mandelate dehydrogenase; $4, P$. putida $\mathrm{L}(+)$-mandelate dehydrogenase; 5, A. calcoaceticus L(+)-mandelate dehydrogenase; 6, A. calcoaceticus $\mathrm{L}(+)$-lactate dehydrogenase.

is a particular advantage in the yeast mandelate dehydrogenase having two functional domains or whether it is simply a consequence of its evolutionary history, remains an open question, especially in view of the fact that there is no experimental evidence as to why the bacterial and yeast $\mathrm{L}(+)$-lactate dehydrogenases should be one- and two-domain enzymes, respectively.
3. The $\mathrm{D}(-)$-mandelate and $\mathrm{D}(-)$-lactate dehydrogenases of $A$. calcoaceticus and the D(-)-lactate dehydrogenase of $E$. coli are probably homologous with each other but not with the $\mathrm{L}(+)$-enantiomer-specific enzymes.

4. Much more work needs to be done to determine whether the bacterial and yeast enzymes with NADlinked $\mathrm{D}(-)$-mandelate dehydrogenase activity are related to each other.

5. The classification of lactate and mandelate dehydrogenases that was proposed previously (Fewson, 1992) can now be revised and extended (Table 3 ) and it has obvious evolutionary implications. This categorization could be refined if enzymes from an even wider range of organisms were to be examined and it introduces the possibility that certain classes of enzyme remain to be discovered [e.g. FAD-containing L(+)-mandelate dehydrogenases, FMN-containing (with or without haem) $\mathrm{D}(-)$-mandelate or $\mathrm{D}(-)$-lactate dehydrogenases, or NAD(P)-dependent $\mathrm{L}(+)$-mandelate dehydrogenases].

6. The suggestion that mandelate dehydrogenases evolved by recruitment of duplicated genes for 2hydroxyacid dehydrogenases (Tsou et al., 1990) is strengthened, as is the view that such evolution occurred on several independent occasions in different organisms and that it exploited the various 2-hydroxyacid dehydrogenases that happened to be available (Fewson, 1992).

Table 3. Types of mandelate dehydrogenases and their relationship to some lactate and other 2-hydroxyacid dehydrogenases

1. NAD(P)-independent

(a) FMN-dependent

(i) FMN, non-haem, $M_{\mathrm{r}}=$ approx. $44000^{*}$

L(+)-Mandelate dehydrogenase of Acinetobacter calcoaceticus

L(+)-Mandelate dehydrogenase of Pseudomonas putida

L(+)-Lactate dehydrogenase of Acinetobacter calcoaceticus

L(+)-Lactate dehydrogenase of Escherichia coli

(ii) FMN, haem, $M_{\mathrm{r}}=$ approx. 59000

$\mathrm{L}(+)$-Mandelate dehydrogenase of Rhodotorula graminis

$\mathrm{L}(+)$-Lactate dehydrogenase of Saccharomyces cerevisiae

$\mathrm{L}(+)$-Lactate dehydrogenase of Hansenula anomala

(b) FAD-dependent, $M_{\mathrm{r}}=$ approx. 60000

D(-)-Mandelate dehydrogenase of Acinetobacter calcoaceticus

D(-)-Lactate dehydrogenase of Acinetobacter calcoaceticus

D( -)-Lactate dehydrogenase of Escherichia coli

2. NADP-dependent

D( -)-Mandelate dehydrogenase of Rhodotorula graminis

D( -)-Mandelate dehydrogenase of Lactobacillus curvatus

$\mathrm{D}(-)$-Hydroxyisocaproate (mandelate) dehydrogenase of Streptococcus faecalis

$\mathrm{D}(-)$-Hydroxyisocaproate dehydrogenase of Lactobacillus casei

* There is circumstantial evidence that the mandelate dehydrogenase of Pseudomonas aeruginosa and

Rhizobium leguminosarum are also in this group. 
7. It is clear that the genes for at least one-and possibly more - lactate dehydrogenases and mandelate dehydrogenases representative of each of the groups listed in Table 3 should be cloned and sequenced.

D.P.B. and R.M.C. acknowledge receipt of research studentships from the UK Science and Engineering Research Council and M.Y. is grateful to the Federal Ministry of Education of the Government of Pakistan for a Central Overseas Training Scholarship. We are grateful to the following for various strains of yeasts: Dr C. R. Hipkin, $\mathrm{Mr}$ I. Watt, Ms E. Scott, Prof. C. Ramirez and Dr E. Slavikova.

\section{References}

Allison, N. \& Fewson, C. A. (1986). Purification and properties of Llactate dehydrogenase from Acinetobacter calcoaceticus. FEMS Microbiology Letters 36, 183-186.

Allison, N., O'Donnell, M. J. \& Fewson, C. A. (1985). Membranebound lactate dehydrogenases and mandelate dehydrogenases of Acinetobacter calcoaceticus. Purification and properties. Biochemical Journal 231, 407-416.

BAKER, D. P. \& Fewson, C. A. (1989). Purification and characterization of $\mathrm{D}(-)$-mandelate dehydrogenase from Rhodotorula graminis. Journal of General Microbiology 135, 2035-2044.

Black, M. T., GunN, F. J., Chapman, S. K. \& Reid, G. A. (1989). Structural basis for the kinetic differences between flavocytochromes $b_{2}$ from the yeasts Hansenula anomala and Saccharomyces cerevisiae. Biochemical Journal 263, 973-976.

Campbell, H. D., Rogers, B. L. \& Young, I. G. (1984). Nucleotide sequence of the respiratory D-lactate dehydrogenase gene of Escherichia coli. European Journal of Biochemistry 144, 367-373.

Cederlund, E., Lindquist, Y., Soderlund, G., Branden, C. I. \& JornvalL, H. (1988). Primary structure of glycolate oxidase from spinach. European Journal of Biochemistry 173, 523-530.

Chalmers, R. M., Keen, J. N. \& Fewson, C. A. (1991). Comparison of benzyl alcohol dehydrogenases and benzaldehyde dehydrogenases from the benzyl alcohol and mandelate pathways in Acinetobacter calcoaceticus and from the TOL-plasmid-encoded toluene pathway in Pseudomonas putida. Biochemical Journal 273, 99-107.

Chapman, S. K., White, S. A. \& Reid, G. A. (1991). Flavocytochrome $b_{2}$. Advances in Inorganic Chemistry 36, 257-301.

DURHAM, D. R. (1984). Initial reactions involved in the dissimilation of mandelate by Rhodotorula graminis. Journal of Bacteriology $\mathbf{1 6 0}$, 778-780.

Durham, D. R., MCNamee, D. G. \& Stewart, D. B. (1984). Dissimilation of aromatic compounds in Rhodotorula graminis: biochemical characterisation of pleiotropically negative mutants. Journal of Bacteriology 160, 771-777.

FEwson, C. A. (1988). Microbial metabolism of mandelate: a microcosm of diversity. FEMS Microbiology Reviews 54, 85-110.

Fewson, C. A. (1992). Microbial metabolism and mandelate: occurrence, properties, and evolution of mandelate dehydrogenases and other enzymes of the mandelate pathway. In The Evolution of Metabolic Function, pp. 115-141. Edited by R. P. Mortlock. Boca Raton: CRC Press.

Fewson, C. A., Allison, N., Hamilton, I. D., Jardine, J. \& Scott, A. J. (1988). Comparison of mandelate dehydrogenases from various strains of Acinetobacter calcoaceticus: similarity of natural and 'evolved' forms. Journal of General Microbiology 134, 967-974.

GARVIE, E. I. (1980). Bacterial lactate dehydrogenases. Microbiological Reviews 44, 106-139.
Guiard, B. (1985). Structure, expression and regulation of a nuclear gene encoding a mitochondrial protein: the yeast $\mathrm{L}(+)$-lactate cytochrome $c$ oxidoreductase (cytochrome $b_{2}$ ). EMBO Journal 4, $3265-3272$.

Hegeman, G. D., Rosenberg, E. Y. \& Kenyon, G. L. (1970). Mandelate racemase from Pseudomonas putida. Purification and properties of the enzyme. Biochemistry 9, 4029-4036.

Hoey, M. E. \& Fewson, C. A. (1990). Is there a mandelate enzyme complex in Acinetobacter calcoaceticus or Pseudomonas putida? Journal of General Microbiology 136, 219-226.

Hoey, M. E., Allison, N., Scott, A. J. \& Fewson, C. A. (1987). Purification and properties of L-mandelate dehydrogenase and comparison with other membrane-bound dehydrogenases from Acinetobacter calcoaceticus. Biochemical Journal 248, 871-876.

Hummel, W., SChÜTTE, H. \& Kula, M.-R. (1988). D(-)-Mandelic acid dehydrogenase from Lactobacillus curvatus. Applied Microbiology and Biotechnology 28, 433-439.

Jamaluddin, M., Subba Rao, P. V. \& Vaidyanathan, C. S. (1970). Involvement of the protocatechuate pathways in the metabolism of mandelic acid by Aspergillus niger. Journal of Bacteriology 101, 786-793.

Kishore, S., Sugumaran, M. \& Vaidyanathan, C. S. (1976), Metabolism of D,L- $( \pm)$-phenylalanine by Aspergillus niger. Journal of Bacteriology 128, 182-191.

Lederer, F., Cortial, S., Becam, A.-M., Haumont, P.-Y. \& Perez, L. (1985). Complete amino acid sequence of flavocytochrome $b_{2}$ from baker's yeast. European Journal of Biochemistry 152, 419-428.

Lerch, H.-P., Blocker, H., Kallwas, H., Hoppe, J., Tsai, H. \& Collins, J. (1989). Cloning, sequencing and expression in Escherichia coli of the D-2-hydroxyisocaproate dehydrogenase gene of Lactobacillus casei. Gene 78, 47-57.

Ramakrishna RaO, D. N. \& Vaidyanathan, C. S. (1977). Metabolism of mandelic acid by Neurospora crassa. Canadian Journal of Microbiology 23, 1496-1499.

Ramanarayanan, M. \& Vaidyanathan, C. S. (1973). Mandelate oxidase of Aspergillus niger. Part I. Properties of particulate D $(-)$ mandelate oxidase. Indian Journal of Biochemistry and Biophysics 10, 254-256.

Rule, G. S., Pratt, E. A., Chin, C. C. Q., Wold, F. \& Ho, C. (1985). Overproduction and nucleotide sequence of the respiratory $\mathrm{D}(-)$ lactate dehydrogenase of Escherichia coli. Journal of Bacteriology 161, 1059-1068.

TAGUCHI, H. \& OHTA, T. (1991). D-Lactate dehydrogenase is a member of the D-isomer-specific 2-hydroxyacid dehydrogenase family. Journal of Biological Chemistry 266, 12588-12594.

Tsou, A. Y., Ransom, S. C., Gerlt, J. A., Buechter, D. D., Babbitt, P. C. \& KenYon, G. L. (1990). Mandelate pathway of Pseudomonas putida: sequence relationships involving mandelate racemase, $(S)$ mandelate dehydrogenase, and benzoylformate decarboxylase and expression of benzoylformate decarboxylase in Escherichia coli. Biochemistry 29, 9856-9862.

Volokita, M. \& Somerville, C. R. (1987). The primary structure of spinach glycolate oxidase from the DNA sequence of a cDNA clone. Journal of Biological Chemistry 262, 15825-15828.

Wilson, K. J. \& YUAN, P. M. (1989). Protein and peptide purification. In Protein Sequencing: A Practical Approach, pp. 1-41. Edited by J. B. C. Findlay \& M. J. Geisow. Oxford: IRL. Press.

YAMAZAKI, Y. \& MAEDA, H. (1986). Enzymatic synthesis of optically pure $(R)-(-)$-mandelic acid and other 2-hydroxycarboxylic acids: screening for the enzyme, and its purification, characterization and use. Agricultural and Biological Chemistry 50, 2621-2631.

Yasin, M. \& Fewson, C. A. (1993). L(+)-Mandelate dehydrogenase from Rhodotorula graminis: purification, partial characterization and identification as a flavocytochrome b. Biochemical Journal (in the Press). 